\title{
Comparative Study of MELD Score and Glasgow Coma Scale in Patients with Hepatic Encephalopathy
}

\author{
Samir Mohamed Kabil, Magdy Abdel-Mawgood Gad, \\ Naglaa El-Toukhy Ramadan El-Toukhy, Nahed Samir Abdel-Moteleb \\ Hepatology, Gastroenterology and Infectious Diseases Department, Faculty of Medicine, \\ Benha University,Benha, Egypt.
}

Corresponding Author Nahed S Abdel-Motelb

Mobile:+2010973387
85
E
mail:dr_n33@hotmail.
com

Key words: Hepatic encephalopathy, GCS, MELD score, uMELD score, Child score
Background and study aim: Hepatic encephalopathy occurs in approximately $30-45 \%$ of patients with cirrhosis and $10-$ $50 \%$ of patients with transjugular intrahepatic Porto systemic shunt, while minimal hepatic encephalopathy affects approximately 20$60 \%$ of patients with liver disease. There are multiple prognostic scores that predict the mortality from chronic liver disease, of which the Child-Pugh score and the Model for End-stage Liver Disease (MELD) score are the most commonly used. The most widely used scale used to evaluate HE is the West-Haven (WH) scale, with scores ranging from 0 to 4 . This study is designed to compare Glasgow coma scale to West-Haven scale in prediction of prognosis and survival of patients with hepatic encephalopathy.

\section{INTRODUCTION}

Hepatic encephalopathy (HE) is a serious complication of decompensated cirrhosis that manifests as a wide range of neuropsychological clinical findings ranging from minimal $\mathrm{HE}$ to coma [1]. HE can be classified as either 'overt' or 'minimal'. Overt $\mathrm{He}(\mathrm{oHE})$ is a syndrome of neurological and neuronpsychiatric abnormalities that can be detected by bedside clinical tests. By contrast, patients with minimal He (mHE) present with normal mental and neurological status upon clinical examination but specific psychometric tests yield abnormal results [2]. Despite the important progresses of neuron imaging methods, clinical scales are commonly considered the best way to assess the degree of impairment and its impact on daily life activities for the majority of neurological diseases.
Patients and Methods: This study was conducted on 100 patients with liver cirrhosis and overt Hepatic Encephalopathy admitted to The Department of Hepatology, Gastroenterology and Infectious Diseases of Mansoura Health Insurance Hospital, divided into four groups according to the grade of encephalopathy by West-Haven Criteria.

Results: There was no difference in prediction of survival among the studied patients assessed by GCS, MELD score, UMELD score and Child score (all had the same results).

Conclusion: Glasgow Coma Scale can be a prognostic tool for morbidity and mortality, as well as, follow-up in patients with $\mathrm{HE}$ and.

Previous studies recommended using clinical scales for grading hepatic encephalopathy and to report efficacy in therapeutic trials such as the WestHaven criteria and Glasgow Coma Scale to assess the severity of HE [3].

The model for end-stage liver disease (MELD) score was introduced to evaluate hepatic functions in cirrhotic patients. It has the advantage of using three objective and easily measured parameters: creatinine levels, international normalized ratio (INR) and total bilirubin [4]. The Model for End-Stage Liver Disease (MELD) score has been adopted as an objective indicator of liver disease severity [1].

Aim of the work: this study aims at assessing the significance of Glasgow coma scale in evaluation of patients with hepatic encephalopathy in comparison 
to the standard West-Haven criteria and its ability to predict morbidity and mortality in patients with hepatic encephalopathy in comparison with MELD score.

\section{PATIENTS AND METHODS}

This study was carried out on 100 patients with liver cirrhosis and overt Hepatic Encephalopathy. They were 85 males $(85 \%)$ and 15 females $(15 \%)$, and their ages ranged between 18 and 60 years. All cases were selected from the Department of Hepatology, Gastroenterology and Infectious Diseases, Mansoura Health Insurance Hospital, within the period between January 2014 to June 2014.

The exclusion criteria were severe cardio-pulmonary disease, sepsis, renal disease, hepatocellular carcinoma, diabetes mellitus, patients listed to undergo transplantation.

\section{Patients were subjected to the following:}

Full history taking, thorough clinical examination. Routine laboratory investigations, that included: Complete blood picture. Liver profile tests: prothrombin time and concentration S. creatinine, viral markers, arterial blood ammonia.

Samples collection, preparation and handling: A sample of arterial blood was sampled soon after admission under aseptic condition in preheparinized syringes from indwelling radial or femoral arterial catheters. Admission samples were taken within 24 hours of admission. Ammonia was measured with Ammonia Test Kit II for the PocketChem BA device (Arkay, Inc., Kyoto, Japan).

\section{Abdominal Ultrasonography:}

Liver was assessed for: size (span), echogenicity, surface, thickening of portal tracts, portal vein diameter, hepatic veins, inferior vena cava and presence or absence of focal lesions.

Spleen was assessed for: size, echogenicity, splenic vein diameter and presence or absence of collaterals. Other data concerning the gall bladder, both kidneys, pancreas, para aortic region as well as detection of ascites all were fulfilled.

The severity of liver cirrhosis in Hepatic Encephalopathy assessed using: Modified Child score:

Evaluation of the severity of liver cirrhosis was obtained in each cirrhotic patient with modified Child-Pugh score. This system relies on clinical and laboratory evaluation including ascites, grade of encephalopathy, serum albumin, bilirubin and prothrombin time [5].

\begin{tabular}{|l|l|l|l|}
\hline Parameter & 1 & 2 & 3 \\
\hline Ascites controlled & None & easily controlled & Poorly controlled \\
\hline Encephalopathy & none & grades 1-2 & grades 3-4 \\
\hline Bilirubin $(\mathrm{mg} / \mathrm{dl})$ & $<2.0$ & $2-3$ & $>3.0$ \\
\hline Albumin $(\mathrm{g} / \mathrm{dL})$ & $>3.5$ & $2.8-3.5$ & $<2.8$ \\
\hline Prothrombin time (seconds increased) & $<4$ & $4-6$ & $>6$ \\
\hline
\end{tabular}

\begin{tabular}{|c|c|c|c|}
\hline Points & Class & One year survival & Two year survival \\
\hline $5-6$ & A & $100 \%$ & $85 \%$ \\
\hline $7-9$ & B & $81 \%$ & $57 \%$ \\
\hline $10-15$ & C & $45 \%$ & $35 \%$ \\
\hline
\end{tabular}

\section{MELD score:}

(Model for end stage liver disease) for evaluation of the severity of liver cirrhosis in each cirrhotic patient, and this system relies on laboratory evaluation including serum bilirubin, serum creatinine and INR (international normalized ratio).

MELD score $=\{9.6 \times \log ($ creatinine $\mathrm{mg} / \mathrm{dL})+3.8$ $\times \log$ (bilirubin $\mathrm{mg} / \mathrm{dL})+11.2 \times \log (\mathrm{INR})+6.4\}[6]$. 
West Haven Criteria for Grading of mental status in HE [7]:

\begin{tabular}{|c|c|c|c|}
\hline Grade 1 & Grade 2 & Grade 3 & Grade 4 \\
\hline $\begin{array}{l}\text { - Trivial lack of awareness } \\
\text { - Euphoria or anxiety } \\
\text { - Shortened attention span } \\
\text { - Impairment of addition or } \\
\text { subtraction } \\
\text { - Altered sleep rhythm }\end{array}$ & $\begin{array}{l}\text { - Lethargy or apathy } \\
\text { - Disorientation for time } \\
\text { - Obvious personality } \\
\text { change } \\
\text { - Inappropriate behavior } \\
\text { - Dyspraxia } \\
\text { - Asterixis }\end{array}$ & $\begin{array}{l}\text { - Somnolence to semi- } \\
\text { stupor } \\
\text { - Responsive to stimuli } \\
\text { - Confused } \\
\text { - Gross disorientation } \\
\text { - Bizarre behavior } \\
\text { - }\end{array}$ & $\begin{array}{l}\text { Coma with or } \\
\text { without } \\
\text { painful } \\
\text { stimuli } \\
\text { response to }\end{array}$ \\
\hline
\end{tabular}

Glasgow Coma Scale: [8]

\begin{tabular}{|c|c|c|}
\hline Eye Opening Response & Verbal Response & Motor ResponsE \\
\hline $\begin{array}{l}\text { - } \text { Spontaneous-open with } \\
\text { blinking at base line } 4 \\
\text { - To verbal stimuli, } \\
\text { command, speech } 3 \\
\text { - To pain only (not } \\
\text { applied to face) } 2 \\
\text { - No response } 1 \\
\text { - }\end{array}$ & $\begin{array}{l}\text { - Oriented } 5 \\
\text { - Confused conversation, but } \\
\text { able to answer questions } 4 \\
\text { - Inappropriate words } 3 \\
\text { - Incomprehensible speech } \\
2 \\
\text { - No response } 1\end{array}$ & $\begin{array}{l}\text { - Obeys commands for movement } 6 \\
\text { - Purposeful movement to painful } \\
\text { stimulus } 5 \\
\text { - Withdraws in response to pain } 4 \\
\text { - Flexion in response to pain } \\
\text { (decorticate } \\
\text { - posturing) } 3 \\
\text { - Extension response in response to } \\
\text { pain (decerebrate posturing) } 2 \\
\text { - No response } 1\end{array}$ \\
\hline
\end{tabular}

\section{Statistical Analysis}

Data were tabulated, coded then analyzed using the computer program SPSS (Statistical package for social science) version 21 to obtain Descriptive statistics were calculated in the form of: A- Mean \pm Standard deviation (SD) for quantitative parametric data. B- Median and range (Minimum - maximum) for quantitative non-parametric data. C- Frequency (Numberpercent) for qualitative data.

In the statistical comparison between the different groups, the significance of difference was tested using one of the following tests :

A-Student's $t$-test:-Used to compare between mean of two groups of numerical (parametric) data. B- Mann Whitney U test: Used to compare between two groups of numerical (nonparametric) data. C- Kruskal Wallis test: Used to compare between more than two groups of numerical (non-parametric) data followed by Mann Whitney for multiple comparisons.

Significance level: For all above mentioned statistical tests done, the threshold of significance is fixed at 5\% level (p-value). The results were considered: Non-significant when the probability of error is more than 5\% ( $p>0.05)$. Significant when the probability of error is less than $5 \%$ $(\mathrm{p} \leq 0.05)$. Highly significant when the probability of error is less than $0.1 \%(\mathrm{p} \leq 0.001)$.

\section{RESULTS}

The study was conducted on 100 patients (cases group) 85 males (85\%), 15 females (15\%). the age ranged between 47 and 77 years old in group (1) cases with the mean age being $60.28 \pm 7.54$ years in comparison with group (4), the age ranged between 50 and 66 years old with mean age 56.50 \pm 6.95 years. Table (1) All patients complained from disturbed consciousness.

The mean value of Serum Creatinine was significantly higher in group (3) group than in other groups. No statistical significant difference between the four groups as regards ALT, AST, alkaline phosphatase, total bilirubin, prothrombin concentration, INR and albumin (Table 2).

As shown in table (2) there was statistical significant difference between the four groups as regards the arterial blood ammonia. It was significantly more predominant in group (4) cases (356.50 \pm 47.93$)$ than in group (1) cases (90.73 \pm 18.42$)$.

As shown in table (3) The most cases of Child A were in group (2), most cases of Child B were in 
group (1), and most cases of Child $\mathrm{C}$ were in group (3) with statistical significant difference between the four groups. The mean value of Child score was predominant in group (3) cases $(10 \pm 1.087)$ with highly statistical significant difference between the four groups. As regards the severity of liver disease, MELD score was

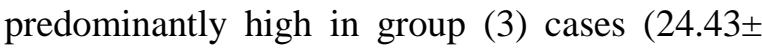
6.45 ) in comparison with group (1) patients $(18.52 \pm 4.62)$. Also uMELD score was high in group (3) cases with statistically significant difference between the four groups. There was statistical significant difference between the four groups as regards the severity of neurological dysfunction assessed by Glasgow Coma Scale. The GCS was predominantly high in group (1) cases, and less in group (4) cases.

As shown in table (4) average cirrhotic liver was detected in $78.9 \%$ of group (1) cases in comparison with group (4) cases $(25.0 \%)$. Enlarged cirrhotic liver was detected predominant in group (4) cases, while shrunken liver was present predominant in group (3) cases and was statistically significant. Splenomegaly was detected in $100 \%$ of group (13-4) cases compared to $97.1 \%$ of group (2) cases which was statistically not significant. Portal Vein Dilatation was detected predominant in group (4) cases (100\%) in comparison with group (1) cases which was present in $(63.1 \%)$ and was statistically significant. There was no statistically significant difference among the four groups as regards the portal vein dilatation, splenic vein dilatation, collaterals, and gall bladder. There was statistical significant difference between the four groups as regards ascites. Mild ascites was significantly more predominant in group (4) patients $(50.0 \%)$ than in other groups. Moderate ascites was significantly more predominant in group (1) patients (63.2\%) than in other groups. Severe ascites was significantly more predominant in group (3) patients (56.5\%) than in other groups.
As shown in table (5) there was statistically significant difference as regards the mortality among the studied patients being more predominant in group (3) patients $(87 \%$ ) in comparison with group (1) cases (34.2\%). West Haven Criteria had significant influence on overall survival of patients with hepatic encephalopathy .There was a longest survival time (mean 8.9 months) in the group (1), followed by a longer survival time (mean 4.13 months) in the group (2) and a shorter survival time (mean 2.8 months) in the group (4) and the shortest survival time (mean 2.11months) in the group (3) which was statistically significant. Table (6)

GCS had significant influence on overall survival of patients with hepatic encephalopathy. After one year, survival was predominant in score $(11.76 \pm 1.93)$ and death in score $(9.24 \pm 2.95)$. MELD score had significant influence on overall survival of patients with HE. After one year, survival was predominantly high when MELD score was early $(17.64 \pm 4.29)$ and death occurred when the score was advanced (23.10 \pm 6.52$)$. Also uMELD score affected on overall survival of patients with HE. After one year, survival was high when uMELD score was early (3.94 \pm .489$)$

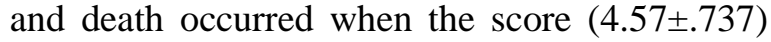
was advanced with high significant statistically difference. Child score affected on overall survival of patients with HE. Survival was high when score was small $(8.38 \pm 1.04)$ and death occurred when the score increased $(9.18 \pm 1.27)$ with significant statistically difference between all groups. Table (7)

As shown in table (8) according to Cox regression, there was no difference in prediction of survival among the studied patients assessed by GCS, MELD score, uMELD score and Child score (all had the same results). 
Table (1): Demographic features of the studied patients

\begin{tabular}{|c|c|c|c|c|c|c|c|c|c|}
\hline Items & \multicolumn{2}{|c|}{$\begin{array}{l}\text { Group } 1 \\
(\mathrm{No}=38) \\
\end{array}$} & \multicolumn{2}{|c|}{$\begin{array}{l}\text { Group } 2 \\
(\mathrm{No}=35) \\
\end{array}$} & \multicolumn{2}{|c|}{$\begin{array}{c}\text { Group } 3 \\
(\text { No23 ) }\end{array}$} & \multicolumn{2}{|c|}{$\begin{array}{c}\text { Group } 4 \\
(\mathrm{No}=4)\end{array}$} & $\begin{array}{c}\text { Test of sig. } \\
\text { p-value }\end{array}$ \\
\hline \multicolumn{10}{|l|}{ Age } \\
\hline Mean \pm SD & \multicolumn{2}{|c|}{$60.28 \pm 7.54$} & \multicolumn{2}{|c|}{$59.22 \pm 8.24$} & \multicolumn{2}{|c|}{$58.69 \pm 9.74$} & \multicolumn{2}{|c|}{$56.50 \pm 6.95$} & $F=.366$ \\
\hline Range & \multicolumn{2}{|c|}{$47-77$} & \multicolumn{2}{|c|}{$45-81$} & \multicolumn{2}{|c|}{$41-86$} & \multicolumn{2}{|c|}{$50-66$} & $\mathrm{P}=.777$ \\
\hline \multicolumn{10}{|l|}{ Sex } \\
\hline Male & 31 & 81.6 & 28 & 80.0 & 22 & 95.7 & 4 & 100 & \multirow{2}{*}{$\begin{array}{l}\mathrm{X} 2=3.788 \\
\mathrm{P}=.285\end{array}$} \\
\hline Female & 7 & 18.4 & 7 & 20.0 & 1 & 4.3 & 0 & 0 & \\
\hline
\end{tabular}

Table (2): Liver and renal function of the studied patients

\begin{tabular}{|c|c|c|c|c|c|}
\hline \multirow[t]{2}{*}{ Items } & $\begin{array}{c}\text { Group } 1 \\
(n=38) \\
\end{array}$ & $\begin{array}{c}\text { Group } 2 \\
(n=35) \\
\end{array}$ & $\begin{array}{c}\text { Group 3 } \\
(n=23) \\
\end{array}$ & $\begin{array}{c}\text { Group4 } \\
(n=4)\end{array}$ & \multirow{2}{*}{$\begin{array}{c}\text { Test of sig. } \\
\text { p-value }\end{array}$} \\
\hline & Mean \pm SD & Mean \pm SD & Mean \pm SD & Mean \pm SD & \\
\hline $\begin{array}{l}\text { Creatinine } \\
\mathrm{mg} / \mathrm{dL}\end{array}$ & $1.17 \pm .659$ & $1.82 \pm 1.42$ & $2.36 \pm 1.60$ & $1.98 \pm 1.55$ & $\begin{array}{l}\mathrm{P} 1=.030 \\
\mathrm{p} 2=.001 \\
\mathrm{p} 3=.222 \\
\mathrm{p} 4=.108 \\
\mathrm{p} 5=.805 \\
\mathrm{p} 6=.574\end{array}$ \\
\hline $\begin{array}{l}\text { Albumin } \\
\mathrm{gm} / \mathrm{dL}\end{array}$ & $2.70 \pm .402$ & $2.69 \pm .444$ & $2.502 \pm .407$ & $2.58 \pm .576$ & $\begin{array}{l}\mathrm{F}=1.304 \\
\mathrm{P}=.278\end{array}$ \\
\hline $\begin{array}{l}\text { Alkaline } \\
\text { Phosphatase }\end{array}$ & $178.26 \pm 48.41$ & $177.31 \pm 79.18$ & $185.61 \pm 43.02$ & $196 \pm 24.06$ & $\begin{array}{l}F=.197 \\
P=.898\end{array}$ \\
\hline ALT & $45.60 \pm 21.41$ & $39.91 \pm 20.55$ & $56.47 \pm 48.45$ & $48.50 \pm 23.17$ & $\begin{array}{l}\mathrm{F}=1.455 \\
\mathrm{p}=.232\end{array}$ \\
\hline AST & $47.02 \pm 20.37$ & $51.25 \pm 21.69$ & $72.17 \pm 64.68$ & $41 \pm 18.07$ & $\begin{array}{l}F=2.671 \\
p=.052\end{array}$ \\
\hline $\begin{array}{l}\text { Bilirubin } \\
\mathrm{mg} / \mathrm{dl}\end{array}$ & $4.87 \pm 3.51$ & $5.11 \pm 3.82$ & $5.81 \pm 3.22$ & $3.42 \pm .783$ & $\begin{array}{l}F=.674 \\
p=.570\end{array}$ \\
\hline $\begin{array}{l}\text { Prothrombin } \\
\text { conc }\end{array}$ & $16.26 \pm 3.06$ & $16.42 \pm 3.31$ & $17.64 \pm 4.09$ & $15.17 \pm .899$ & $\begin{array}{l}F=1.136 \\
P=.338\end{array}$ \\
\hline INR & $1.61 \pm .45$ & $1.59 \pm .526$ & $1.66 \pm .494$ & $1.53 \pm .147$ & $\begin{array}{l}\mathrm{F}=.131 \\
\mathrm{P}=.942\end{array}$ \\
\hline $\begin{array}{l}\text { Arterial Blood } \\
\text { Ammonia } \\
\text { mg/dl }\end{array}$ & $90.73 \pm 18.42$ & $123.28 \pm 27.53$ & $245.47 \pm 60.26$ & $356.50 \pm 47.93$ & $\begin{array}{l}\mathbf{P} 1=.000 * * \\
\text { p2 }=.000 * * \\
\text { p3 }=.000 * * \\
\text { p4 }=.000 * * \\
\text { p5 }=.000 * * \\
\text { p6 }=.000 * *\end{array}$ \\
\hline
\end{tabular}

P1 comparison between groups $1-2$. P2 comparison between groups $1-3$. P3 comparison between groups $1-$ 4. P4 comparison between groups 2 - 3. P5 comparison between groups 2 - 4. P6 comparison between groups 3 -4 . $^{*}$ significant, ${ }^{* *}$ highly significant 
Table (3): The severity of liver cirrhosis assessed by Child- Pugh classification, MELD and Glasgow Coma Scale

\begin{tabular}{|c|c|c|c|c|c|c|c|c|c|}
\hline \multirow{2}{*}{ Items } & \multicolumn{2}{|c|}{ Group 1} & \multicolumn{2}{|c|}{ Group 2} & \multicolumn{2}{|c|}{ Group 3} & \multicolumn{2}{|c|}{ Group 4} & \multirow{2}{*}{$\begin{array}{c}\text { Test of sig. } \\
\text { p-value }\end{array}$} \\
\hline & $\mathrm{No}=38$ & $\% 100$ & No $=35$ & $\% 100$ & $\mathrm{No}=23$ & $\% 100$ & No $=4$ & $\% 100$ & \\
\hline \multicolumn{10}{|c|}{ Child grade } \\
\hline Child A & 1 & 2.6 & 1 & 2.9 & 0 & 0 & 0 & 0 & \multirow{3}{*}{$\begin{array}{l}\text { P1 }=.985 \\
\text { p2 }=.000 * * \\
\text { p3 }=.857 \\
\text { p4 }=.001 * * \\
\text { p5 }=.883 \\
\text { p6 }=.273\end{array}$} \\
\hline Child B & 31 & 81.6 & 28 & 80.0 & 8 & 34.8 & 3 & 75.0 & \\
\hline Child C & 6 & 15.8 & 6 & 17.1 & 15 & 65.2 & 1 & 25.0 & \\
\hline \multicolumn{10}{|c|}{ Child score } \\
\hline $\begin{array}{l}\text { Mean } \quad \pm \\
\text { SD }\end{array}$ & \multicolumn{2}{|c|}{$8.50 \pm 1.059$} & \multicolumn{2}{|c|}{$8.48 \pm 1.14$} & \multicolumn{2}{|c|}{$10 \pm 1.087$} & \multicolumn{2}{|c|}{$9.25 \pm .50$} & $\begin{array}{l}\text { P1 }=.955 \\
\text { p2 }=.000 * * \\
\text { p3 }=.192 \\
\text { p4 }=.000 * * \\
p 5=.185 \\
p 6=.205\end{array}$ \\
\hline $\begin{array}{l}\text { MELD } \\
\text { score }\end{array}$ & \multicolumn{2}{|c|}{$18.52 \pm 4.62$} & \multicolumn{2}{|c|}{$21.34 \pm 6.92$} & \multicolumn{2}{|c|}{$24.43 \pm 6.45$} & \multicolumn{2}{|c|}{$21 \pm 6.37$} & $\begin{array}{l}\text { P1 }=.048 \\
\text { p2 }=.000 * * \\
\text { p3 }=.435 \\
\text { p4 }=.058 \\
\text { p5 }=.913 \\
\text { p6 }=.293\end{array}$ \\
\hline $\begin{array}{l}\text { uMELD } \\
\text { score }\end{array}$ & \multicolumn{2}{|c|}{$4.08 \pm .589$} & \multicolumn{2}{|c|}{$4.34 \pm .785$} & \multicolumn{2}{|c|}{$4.73 \pm .674$} & \multicolumn{2}{|c|}{$4.22 \pm .618$} & $\begin{array}{l}\text { P1 }=.103 \\
\text { p2 }=.001 * * \\
\text { p3 }=.691 \\
\text { p4 }=.039 * \\
\text { p5 }=.739 \\
\text { p6 }=.176\end{array}$ \\
\hline GCS & \multicolumn{2}{|c|}{$12.84 \pm 0.369$} & \multicolumn{2}{|c|}{$10.62 \pm 0.91$} & \multicolumn{2}{|c|}{$6.43 \pm 0.843$} & \multicolumn{2}{|c|}{$3.75 \pm 1.50$} & $\begin{array}{l}\mathbf{P 1}=.000 * * \\
\text { p2 }=.000 * * \\
\text { p3 }=.000 * * \\
\text { p4 }=.000 * * \\
\text { p5 }=.000 * * \\
\text { p6 }=.000 * *\end{array}$ \\
\hline
\end{tabular}

P1 comparison between groups $1-2$. P2 comparison between groups $1-3$. P3 comparison between groups $1-$ 4. P4 comparison between groups $2-3$. P5 comparison between groups $2-4$. P6 comparison between groups 3 $-4 .{ }^{*}$ significant, ${ }^{* *}$ highly significant 
Table (4): Ultrasonographic features of the studied patients

\begin{tabular}{|c|c|c|c|c|c|c|c|c|c|}
\hline Items & \multicolumn{2}{|c|}{$\begin{array}{l}\text { Group } 1 \\
(\mathrm{No}=38) \\
\end{array}$} & \multicolumn{2}{|c|}{$\begin{array}{l}\text { Group } 2 \\
(\text { No=35 }) \\
\end{array}$} & \multicolumn{2}{|c|}{$\begin{array}{l}\text { Group } 3 \\
(\mathrm{No}=23)\end{array}$} & \multicolumn{2}{|c|}{$\begin{array}{c}\text { Group } 4 \\
(\mathrm{No}=4)\end{array}$} & $\begin{array}{c}\text { Sig. } \\
\text { P }\end{array}$ \\
\hline \multicolumn{10}{|l|}{ Liver size } \\
\hline Average & 30 & 78.9 & 27 & 77.1 & 7 & 30.4 & 1 & 25.0 & \multirow{3}{*}{$\begin{array}{l}\text { P1 }=.983 \\
\text { p2 }=.001 * * \\
\text { p3 }=.032 \\
\text { p4 }=.002 * \\
\text { p5 }=.045 * \\
\text { p6 }=.346\end{array}$} \\
\hline Enlarged & $\overline{1}$ & 2.6 & 1 & 2.9 & $\overline{1}$ & 4.3 & 1 & 25.0 & \\
\hline Shrunk & 7 & 18.4 & 7 & 20.0 & 15 & 65.2 & 2 & 50.0 & \\
\hline \multicolumn{10}{|c|}{ Splenomegaly } \\
\hline Remove & 0 & 0 & 1 & 2.9 & 0 & 0 & 0 & 0 & \multirow{2}{*}{$\begin{array}{l}X^{2}=1.876 \\
P=.599\end{array}$} \\
\hline $\begin{array}{l}\text { Enlarged } \\
(>13 \mathrm{~cm})\end{array}$ & 38 & 100 & 34 & 97.1 & 23 & 100 & 4 & $\overline{100}$ & \\
\hline \multicolumn{10}{|c|}{ Collaterals } \\
\hline No & 11 & 28.9 & 6 & 17.1 & 6 & 26.1 & 1 & 25.0 & \multirow{2}{*}{$\begin{array}{l}\mathrm{X}^{2}=1.469 \\
\mathrm{P}=.689\end{array}$} \\
\hline Yes & 27 & 71.1 & 29 & 82.9 & 17 & 73.9 & 3 & 75.0 & \\
\hline \multicolumn{10}{|l|}{ Ascites } \\
\hline Mild & 2 & 5.3 & 3 & 8.6 & 1 & 4.3 & 2 & 50.0 & \multirow{3}{*}{$\begin{array}{l}\text { P1 }=.324 \\
\text { p2 }=.155 \\
\text { p3 }=.014 \\
\text { p4 }=.663 \\
\text { p5 }=.064 \\
\text { p6 }=.027 *\end{array}$} \\
\hline Moderate & 24 & 63.2 & 16 & 45.7 & 9 & 39.1 & $\overline{1}$ & 25.0 & \\
\hline Severe & 12 & 31.6 & 16 & 45.7 & 13 & 56.5 & 1 & 25.0 & \\
\hline \multicolumn{10}{|l|}{ GB } \\
\hline Thin wall & 10 & 26.3 & 4 & 11.4 & 4 & 17.4 & 0 & 0 & \multirow{3}{*}{$\begin{array}{l}X^{2}=5.553 \\
P=.475\end{array}$} \\
\hline Thick & 27 & 71.1 & 31 & 88.6 & 19 & 82.6 & 4 & 100 & \\
\hline Removed & 1 & 2.6 & 0 & 0 & 0 & 0 & 0 & 0 & \\
\hline
\end{tabular}

P1 comparison between groups 1-2. P2 comparison between groups 1-3. P3 comparison between groups 1-4. P4 comparison between groups 2-3. P5 comparison between groups 2-4. P6 comparison between groups 3-4. significant, ${ }^{* *}$ highly significant

Table (5): One year mortality among the studied patients

\begin{tabular}{|c|c|c|c|c|c|c|c|c|c|}
\hline \multirow{2}{*}{ Survival } & \multicolumn{2}{|c|}{$\begin{array}{c}\text { Group } 1 \\
(n=38)\end{array}$} & \multicolumn{2}{|c|}{$\begin{array}{c}\underset{(n=35)}{\text { Group } 2} \\
(n+1)\end{array}$} & \multicolumn{2}{|c|}{$\begin{array}{c}\text { Group 3 } \\
(n=23)\end{array}$} & \multicolumn{2}{|c|}{$\begin{array}{c}\text { Group } 4 \\
(n=4)\end{array}$} & \multirow{2}{*}{$\begin{array}{l}\text { Test of sig. } \\
\text { p-value }\end{array}$} \\
\hline & No & $\%$ & $\overline{\text { No }}$ & $\%$ & $\overline{\text { No }}$ & $\%$ & No & $\%$ & \\
\hline Survived & 25 & 65.8 & 10 & 28.6 & 3 & $\overline{13.0}$ & 1 & 25.0 & $P 1=.001$ \\
\hline Died & 13 & 34.2 & 25 & 71.4 & 20 & 87.0 & 3 & 75.0 & $\begin{array}{l}\text { p3 }=.146 \\
\text { p4 }=.165 \\
\text { p5 }=1 \\
\text { p6 }=.495\end{array}$ \\
\hline
\end{tabular}


Table (6): Means and Medians for Survival Time of patients with hepatic encephalopathy with reference to West Haven Criteria

\begin{tabular}{|c|c|c|c|c|c|c|}
\hline \multirow{3}{*}{$\begin{array}{c}\text { West } \\
\text { Haven } \\
\text { criteria }\end{array}$} & \multicolumn{4}{|c|}{ Median Survival Time } & \multirow{3}{*}{$\begin{array}{l}\text { Chi- } \\
\text { Square }\end{array}$} & \multirow{3}{*}{ p-value } \\
\hline & \multirow{2}{*}{ Estimate } & \multirow{2}{*}{$\begin{array}{c}\text { Std. } \\
\text { Error }\end{array}$} & \multicolumn{2}{|c|}{ 95\% Confidence Interval } & & \\
\hline & & & Lower Bound & Upper Bound & & \\
\hline 1 & 8.940 & 0.764 & 7.444 & 10.437 & \multirow[t]{5}{*}{32.497} & \multirow[t]{5}{*}{$\leq 001 * *$} \\
\hline 2 & 4.130 & 0.874 & 2.418 & 5.843 & & \\
\hline 3 & 2.119 & 0.851 & 0.451 & 3.787 & & \\
\hline 4 & 2.808 & 2.365 & 0.000 & 7.443 & & \\
\hline Overall & 5.454 & 0.554 & 4.368 & 6.541 & & \\
\hline
\end{tabular}

highly significant

Table (7): Overall survival of patients with hepatic encephalopathy with reference to GCS, MELD score, uMELD score and Child score.

\begin{tabular}{|c|c|c|c|}
\hline & Survived 1 year & Died 1 year & $\begin{array}{c}\text { Test of sig. } \\
\mathbf{p} \text {-value }\end{array}$ \\
\hline GCS & $11.76 \pm 1.93$ & $9.24 \pm 2.95$ & $\begin{array}{c}\mathrm{t}=4.713 \\
\mathbf{p}=\mathbf{. 0 0 0} * *\end{array}$ \\
\hline MELD score & $17.64 \pm 4.29$ & $23.10 \pm 6.52$ & $\begin{array}{c}\mathrm{t}=4.621 \\
\mathbf{p}=.000 * *\end{array}$ \\
\hline $\begin{array}{c}\mathrm{t}=4.691 \\
\text { uMELD }\end{array}$ & $3.94 \pm .489$ & $4.57 \pm .737$ & $\begin{array}{c}\mathrm{p}=\mathbf{. 0 0 0} * * \\
\mathrm{t}=3.268 \\
\mathbf{p}=\mathbf{. 0 0 1} * *\end{array}$ \\
\hline Child score & $8.38 \pm 1.04$ & $9.18 \pm 1.27$ & \\
\hline
\end{tabular}

Table (8): Cox regression for prediction of survival among the studied patients.

\begin{tabular}{|l|c|c|c|c|}
\hline \multirow{2}{*}{ Covariates } & \multirow{2}{*}{ p-value } & \multirow{2}{*}{ Hazard ratio } & \multicolumn{2}{c|}{ 95\% CI for HR } \\
\cline { 3 - 5 } & & & Lower & Upper \\
\hline GCS & $.000^{* *}$ & .762 & .697 & .834 \\
\hline MELD score & $.000^{* *}$ & 1.106 & 1.061 & 1.153 \\
\hline uMELD score & $.000^{* *}$ & 2.412 & 1.666 & 3.491 \\
\hline Child score & $.000^{* *}$ & 1.513 & 1.219 & 1.878 \\
\hline
\end{tabular}

highly significant 


\section{DISCUSSION}

Hepatic encephalopathy is a spectrum of neuropsychiatric manifestations ranging from psychomotor difficulties to altered consciousness and even coma [9]. Hepatic encephalopathy, a challenging complication of advanced liver disease, occurs in approximately $30-45 \%$ of patients with cirrhosis and10-50\% of patients with transjugular intrahepatic Porto systemic shunt, while minimal hepatic encephalopathy affects approximately $20-60 \%$ of patients with liver disease [10]. Minimal hepatic encephalopathy, which is characterized by subtle motor and cognitive deficits, affects approximately $20-60 \%$ of patients with liver disease $[\mathbf{1 1}, \mathbf{1 2}]$. There are multiple prognostic scores that predict the mortality from chronic liver disease, of which the Child-Pugh score and the Model for End-stage Liver Disease (MELD) score are the most commonly used [13]. The MELD score had discriminative ability for 3-month survival of greater than $80 \%$, regardless of the severity of liver disease, without any significant improvement by adding etiology or complications of cirrhosis [6]. MELD is a composite of the patient's laboratory values for serum bilirubin and serum creatinine, and the international normalized ratio (INR) for prothrombin time [13]. The most widely used scale used to evaluate HE is the WestHaven (WH) scale, with scores ranging from 0 to 4. This scale is easy to use but not suitable for patients with altered consciousness and is not well known by physicians other than hepatologists who manage these conditions. For deep coma, the validated Glasgow Coma Scale (GCS) has been proposed [14]. For all these considerations, the main aim of the present study was to assess comparison of MELD score and Glasgow coma scale in prediction of prognosis and survival of patients with hepatic encephalopathy.

In the current study hepatic encephalopathy commonly presented in males more than females. This was in agreement with Mouri et al. [14] and Lehner et al. [15] who reported that men are three times higher than women in most regions.

According to the liver biochemical profile in this study, serum creatinine was the only liver biochemical parameter significantly high in most of patients (group (3) patients. This result was in agreement with Gheorghe et al. [16] and Botta et al. [17] who documented the same results (that in a patient with HE, serum creatinine and INR were the variables significantly associated with six month mortality). In this study, conventional tests of hepatic function did not have statistical significant difference between the four groups, these results were comparable to Botta et al. [17] who documented that exactly. Ammonia has been regarded as one of the major pathogenetic factors of cerebral dysfunction in HE, and astrocyts has been the most commonly affected cell $[\mathbf{1 8 , 1 9 ]}$. Ong et al. [20] showed that venous ammonia levels correlate with the severity of HE. In this study, arterial blood ammonia had a statistical significant difference between the four groups. It was significantly more predominant in

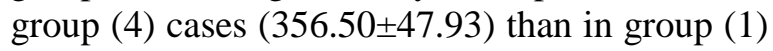
cases $(90.73 \pm 18.42)$. This result was in agreement with Bernal et al. [21] who documented the same results that arterial ammonia on admission was significantly higher in group 4 patients [median: 113-mol/L (74-164-mol/L)], and with Gheorghe et al. [16] who documented that mean plasma ammonia levels were increase with the severity of HE. Our findings support the final report of the working party at the $11^{\text {th }}$ world congresses of gastroenterology ammonia testing was described as a potential diagnostic tool which, however, correlates poorly with symptoms of HE [9].

In this study, most cases of Child A (2.9\%) were in Group (2), most cases of Child B (81.6\%) were in Group (1), and most cases of Child C (65.2) were in Group (3) with statistical significant difference between the four groups. These results were in agreement with Stewart et al. [1] and Botta et al. [17] who documented nearly the same results (63\%) of Group (1) cases were Child B. In contrast, these results disagree with Mouri et al. [14] who reported that most of the patients with Group (1-4) cases were Child $\mathrm{C}(69 \%)$ as he collected patients with severe cirrhosis.

The mean value of Child score was predominantly high in most of patients (group (3) cases) $(10 \pm 1.087)$ with highly statistical significant difference between the four groups. These results were in agreement with Wehler et al. [22] and Botta et al. [17] who documented nearly the same results $(10.9 \pm 1.8)$ in Group (24) cases, Child score was (9-10) in Group (3-4) cases [1], and studies who stated that the mean value of Child score was (9-14) in Group (2-4) cases [17]. D'Amico et al. [23] found that the Child score, albumin, bilirubin, age, ascites, prothrombin time were the most common predictors of survival in patients with HE. 
According to MELD score most of patients (Group (3) patients) $(24.43 \pm 6.45)$ were presented at advanced score comparing to the Group (2) patients (21.34 \pm 6.92$)$ and the Group (4) patients $(21 \pm 6.37)$ which presented at intermediate score, and the early score $(18.52 \pm 4.62)$ were presented in Group (1) patients with statistical significant difference between the four groups. These results in agreement with Stewart et al. [1] who stated that the mean value of MELD score in Group (3) patients were (15-25), in Group (2) patients were (13-22), and in Group (1) patients were (7-17), and Laferrière et al. [24] who documented nearly the same results with a median of $22(17-28)$ in Group (3) patients, and with that who reported that most of the patients with Group (2-4)cases were presented at advanced score $(22 \pm 9)$ and Group (1) cases were presented at early score ( $19 \pm 8$ ) [14].After clinical examination of patients with HE, it was found that as the grades of HE increased, MELD score and Child score were also high [1]. The MELD score reflects liver disease severity, with higher values indicating worse disease [6,25]. Sanyal et al. [26] demonstrated a strong association between MELD score and developing HE as well as HE and mortality. It has recently been suggested that changes in MELD score may be as important as the absolute MELD score in predicting short-term survival [27]. Baseline MELD score has been shown also to be an accurate predictor of 3-month mortality on the wait list in patients with end-stage liver disease, and it was suggested that the accuracy may extend to up to 1 year [28]. According to uMELD score most of patients (Group 3 patients) $(4.73 \pm 0.674)$ were presented at advanced score comparing to the Group (2) patients (4.34 .785) which presented at intermediate score, and the early score $(4.08 \pm .589)$ were presented in Group 1patients with statistical significant difference between the four. These results were in agreement with Craig et al. [29] which documented nearly the same results. In this study, the mean value of Glasgow Coma Scale was predominantly high in group 1 cases (12.84 \pm 0.369$)$, and less in group 4 cases $(3.75 \pm 1.50)$ with statistical significant difference between the four groups. This result was in agreement with Mouri et al. [14] who documented that the mean value of Glasgow Coma Scale was $(14.9 \pm 0.3)$ in Group 1 cases.

Abdominal ultrasonography was done to evaluate the liver status in the studied patients and all of the patients $(100 \%)$ with HE had sonographic evidence of liver cirrhosis. This goes in agreement with Poordad [30] who stated that, all cases of HE frequently coexists with cirrhosis and studies who documented nearly the same results [31]. This was supported also by Said et al. [28] which reported that cirrhosis is present in the vast majority of patients with HE, and Biselli et al. [32] which documented that cirrhosis of the liver was present in $100 \%$ of patients with HE. Average cirrhotic liver was detected in $78.9 \%$ of group 1 cases in comparison with group 4 cases (25.0\%). Enlarged cirrhotic liver was detected predominant in group 4 cases, while shrunken liver was present predominant in group 3 cases and was statistically significant. This goes in agreement with Stewart et al. [1] who documented nearly the same results, and Mouri et al. [14] who reported that most of the patients $(86 \%)$ had average cirrhotic liver. The Homogenous liver was present in $100 \%$ in the four groups, compared to heterogeneous liver which is present in $0 \%$ in the four groups (as HCC was excluded in this study). This goes in agreement with Stewart et al. [1] who documented nearly the same results, and Laferrière et al. [33] who reported that $100 \%$ in the all groups had homogenous liver. In contrast, these results disagree with Mouri et al. [14] who reported that $14 \%$ of cases had heterogeneous liver, and Lehner et al. [15] who reported that $14.5 \%$ of cases had heterogeneous liver. Splenomegaly was detected in $100 \%$ of group (13-4) cases compared to $97.1 \%$ of group (2) cases. These results was in agreement with Stewart et al. [1] who reported nearly the same results, and Lehner et al. [15] who stated that $100 \%$ in the all groups had splenomegaly. Portal Vein Dilatation was detected more in group 4 cases in comparison with group (1) cases which was present in (63.1\%) and was statistically significant. These results were in agreement with Stewart et al. [1] who documented nearly the same results. There was no statistically significant difference among the four groups as regards the portal vein dilatation, splenic vein dilatation, collaterals, and gall bladder. These results are in agreement with Stewart et al. [1] who reported nearly the same results.

After one-year follow up of patients with HE, it was founded that $(61 \%)$ of patients died. These results in agreement with Fichet et al. [34] who reported nearly the same results (54\%) [34], and Gildea et al. [35] who reported nearly that (69\%) of patients died with a median survival of 1 month. Mortality from studies regarding patients with $\mathrm{HE}$ ranged from $33 \%$ to $91 \%$, depending of severity of the underlying disease $[\mathbf{3 6 , 3 7 ]}$. West 
Haven Criteria had significant influence on overall survival of patients with hepatic encephalopathy. The longest survival time (mean 8.9 months) was in the group 1 , followed by a longer survival time (mean 4.13 months) in the group 2and a shorter survival time (mean 2.80 months) in the group (4) and the shortest survival time (mean 2.11months) in the group 3category group .These results in agreement with Mouri et al. [14] which reported nearly the same results.

In the present study, MELD score had significant influence on overall survival of patients with HE. The score was advanced in the most of patients group 3 category group with a shortest survival time, intermediate in the group (2-4) category group with a longer survival time, early in the group 1 with a longest survival time. These results in agreement with Stewart et al. [1] and Laferrière et al. [24] who documented nearly the same results.

In the present study, Glasgow Coma Scale had a significant influence on overall survival of patients with HE, overall survival was shorter in patients with lower score and longer with higher score. This result was in agreement with Mouri et al. [14] who documented the same results.

\section{CONCLUSION}

Glasgow coma scale can help assess patients with hepatic encephalopathy and can with great accuracy assess risk of one year mortality.

Funding: None.

Conflicts of interest: None.

Ethical approval: A written informed consent was taken from all included patients, and the study was approved by the Ethical Committee of our insitution.

\section{REFERENCES}

1. Stewart CI, Malinchoc M, Kim WR, Kamath PS. Hepatic encephalopathy as a predictor of survival in patients with end stage liver disease. Liver transplantation 2007; 13:1366-1371.

2. Prakash R, Mullen KD. Mechanisms, diagnosis and management of HE. Nat Rev Gastroenterol Hepatol 2010;7 (9): 515-25.

3. Ortiz M, Jacas C, Cordoba J: Minimal hepatic encephalopathy. diagnosis, clinical significance and recommendations. J Hepatol 2005;42: S45S53.

4. Ham J, Gish RG, Mullen K, Model for EndStage Liver Disease (MELD). Liver transplantation, 2006;S102-S104.
5. Pugh RN, Murray-Lyon IM, Dawson JL, Pietroni MC, Williams R.Transection of the oesophagus for bleeding oesophageal varices. Br J Surg 1973; 60: $646-649$.

6. Kamath PS, Wiesner RH, Malinchoc M. A model to predict survival in patients with end-stage liver disease. Hepatology 2001;33: 464-470.

7. Mullen KD, Ong JP, Aggarwal A, Krieger D, Easley KA, Karafa MT, Van Lente F, Arroliga AC.Correlation between ammonia levels and the Severity of HE. The American journal of medicine 2003;114: 188 -193.

8. Teasdale G , Jennett B.Assessment of coma and impaired consciousness. Lancet 2003; 81-84.

9. Ferenci P, Lockwood A, Mullen K, Tarter R, Weissenborn K, Blei AT.Hepatic encephalopathydefinition, nomenclature, diagnosis, and quantification: final report of the working party at the $11 \mathrm{~h}$ World Congresses of Gastroenterology, Vienna. Hepatology 2002;35: 716-721.

10. Poordad FF. Review article: the burden of hepatic encephalopathy. Aliment Pharmacol Ther. 2007; 25 Suppl 1: 3-9.

11. Romero-Gomez M, Cordoba J, Jover R, Olmo JA, Ramirez M, Rey R, et al.Value of the critical flicker frequency in patients with minimal hepatic encephalopathy. Hepatology 2007;45:879-885.

12. Das A, Dhiman RK, Saraswat VA, Verma M, Naik SR. Prevalence and natural history of subclinical hepatic encephalopathy in cirrhosis. $J$ Gastroenterol Hepatol 2001; 16(5):531-5.

13. Freeman R.B, Wiesner RH, Edwards E, Harper A, Merion R, Wolfe R.Results of the first year of the new liver allocation plan. Liver Transpl 2004; 10: 7-15.

14. Mouri S, Tripon S, Rudler M, Mallet M, Mayaux J, Thabut D, Weiss N.FOUR Score, a reliable score for assessing overt hepatic encephalopathy in cirrhotic patients. Neurocritical Care 2014; 1541- 6933.

15. Lehner S, Stemmler H, Mück A, Braess J, Parhofer KG. Prognostic parameters and risk stratification in intensive care patients with severe liver diseases. J Gastrointestinal Liver Dis 2014;19(4): 399 -404.

16. Gheorghel L, Iacob S, Iacob R, Gheorghe1C, Popescu I.Variation of the MELD Score as a predictor of death on the waiting list for liver transplantation. $J$ Gastrointestinal Liver Dis 2007; 16 (3) 267-272.

17. Botta F, Giannini E, Romagnoli P, Fasoli A, Malfatti F, Chiarbonello B, et al. MELD scoring system is useful for predicting prognosis in patients with liver cirrhosis and is correlated with residual liver function: a European study. Gut 2003;52:134-139.

18. Bajaj JS. The modern management of hepatic encephalopathy. Aliment Pharmacol Ther 2009; $31: 537-47$. 
19. Bhatia V, Singh R, Acharya SK. Predictive value of arterial ammonia for complications and outcome in acute liver failure. Gut 2006; 55:98104.

20. Ong JP, Aggarwal A, Krieger D, Easley KA, Karafa MT, Van Lente F, et al.Correlation between ammonia levels and the severity of hepatic encephalopathy. Am J Med 2003; 114: 188-193.

21. Bernal W, Hall C, Karvellas C J, Auzinger G, Sizer E, and Wendon J. Arterial Ammonia and Clinical Risk Factors for Encephalopathy and Intracranial Hypertension in Acute Liver Failure. Hepatology 2007;46:1844-1852.

22. Wehler M, Kokoska J, Reulbach U, Hahn EJ and Strauss R: Short-Term Prognosis in Critically Ill Patients with Cirrhosis Assessed by Prognostic Scoring Systems. Hepatology 2001; 34:255-261.

23. D’Amico G, Garcýa-Tsao G, Pagliaro L. Natural history and prognostic indicators of survival in cirrhosis: a systematic review of 118 studies. $J$ Hepatol 2006; 44: 217-231.

24. Laferrière VM, Homberger C, Bichoupan K, Dieterich DT. MELD score and antibiotics use are predictors of length of stay in patients hospitalized with hepatic encephalopathy. BMC Gastroenterology 2014; 14:185.

25. Wiesner R, Edwards E, Freeman R, Harper A, Kim R, Kamath $\mathrm{P}$, et al. Model for end-stage liver disease (MELD) and allocation of donor livers. Gastroenterology 2003; 124: 91-96.

26. Sanyal AJ, Banas C, Sargeant C, Luketic VA, Sterling RK, Stravitz RT, et al. Similarities and differences in outcomes of cirrhosis due to nonalcoholic steatohepatitis and hepatitis C. Hepatology 2006;43:682-689.

27. Saab S, Landaverde C, Ibrahim A, Durazo F, Han S, Yersiz H, Farmer D, Ghobrial R, Goldstein L, Tong M, et al.The MELD Score in Advanced Liver Disease: Association with Clinical Portal Hypertension and Mortality. Experimental and clinical transplantation 2004; 4(1): 395-399.

28. Said A, Williams J, Holden J, Remington P, Gangnon R, Musat A, et al. Model for end stage liver diseases core predicts mortality across abroad spectrum of liver disease. J Hepatol 2004; 40: 897-903.
29. Craig DG, Reid TW, Wright EC, Martin KG, Davidson JS, Hayes PC, Simpson KJ: The sequential organ failure assessment (SOFA) score is prognostically superior to the model for endstage liver disease (MELD) and MELD variants following paracetamol (acetaminophen) overdose. Aliment Pharmacol Ther 2012; 35: 705-713.

30. Poordad FF. Review article: the burden of hepatic encephalopathy. Aliment Pharmacol Ther.2007; 25 Suppl 1: 3-9.

31. Biggins SW, Rodriguez HJ, Bacchetti P, Bass NM, Roberts JP, Terrault NA. Serum sodium predicts mortality in patients listed for liver transplantation. Hepatology 2005,41: 32-39.

32. Biselli M, Gitto S., Gramenzi A. Six score systems to evaluate candidates with advanced cirrhosis for orthotopic liver transplant: which is the winner? .Liver Transpl 2010;16: 964-973.

33. Laferrière VM, Homberger $\mathrm{C}$, Bichoupan $\mathrm{K}$, Dieterich DT. MELD score and antibiotics use are predictors of length of stay in patients hospitalized with hepatic encephalopathy. BMC Gastroenterology 2014; 14:185.

34. Fichet J, Mercier E, Genée O, Garot D, Legras A, Dequin F, Perrotin D. Prognosis and 1-year mortality of intensive care unit patients with severe hepatic encephalopathy. Journal of Critical Care 2009; 24, 364-370.

35. Gildea TR, Cook WC, Nelson DR. Predictors of long-term mortality in patients with cirrhosis of the liver admitted to a medical ICU. Chest 2004; 126: 598-603.

36. Aggarwal A, Ong JP, Younossi ZM. Predictors of mortality and resource utilization in cirrhotic patients admitted to the medical ICU. Chest 2001; 119:1489-97.

37. Benhaddouch Z, Abidi K, Naoufel M. Mortality and prognostic factors of the cirrhotic patients with hepatic encephalopathy admitted to medical intensive care unit. Ann Fr Anesth Reanim 2007; 26: 490-5.

Peer reviewers: Rashed M Hasan, Professor of Tropical Medicine and Hepatogastroenterology, Faculty of Medicine, Zagazig University, Egypt.

Ashraf M Abdelfatah, Assistant Professor of Tropical Medicine and Hepatogastroenterology, Faculty of Medicine, Zagazig University, Egypt. Editor: Mohamad Emara, Assistant Professor of Tropical Medicine and Hepatogastroenterology, Faculty of Medicine, Zagazig University, Egypt. 